\title{
Cribriform architecture in radical prostatectomies predicts oncological outcome in Gleason score 8 prostate cancer patients
}

\author{
Eva Hollemans $\mathbb{D}^{1} \cdot$ Esther I. Verhoef $\mathbb{D}^{1} \cdot$ Chris H. Bangma $^{2} \cdot$ John Rietbergen ${ }^{3}$. Susanne Osanto ${ }^{4}$. \\ Rob C. M. Pelger ${ }^{5}$. Tom van Wezel $\mathbb{1}^{6}$. Henk van der Poel ${ }^{7}$ - Elise Bekers ${ }^{8}$ - Jozien Helleman ${ }^{2}$. \\ Monique J. Roobol $\left(10^{2} \cdot\right.$ Geert J. L. H. van Leenders ${ }^{1}$
}

Received: 5 May 2020 / Revised: 2 July 2020 / Accepted: 2 July 2020 / Published online: 20 July 2020

(c) The Author(s) 2020. This article is published with open access

\begin{abstract}
The Gleason score is an important parameter for clinical outcome in prostate cancer patients. Gleason score 8 is a heterogeneous disease including Gleason score $3+5,4+4$, and $5+3$ tumors, and encompasses a broad range of tumor growth patterns. Our objective was to characterize individual growth patterns and identify prognostic parameters in Gleason score 8 prostate cancer patients. We reviewed 1064 radical prostatectomy specimens, recorded individual Gleason 4 and 5 growth patterns as well as presence of intraductal carcinoma, and evaluated biochemical recurrence- and metastasis-free survival. Gleason score 8 disease was identified in 140 (13\%) patients, of whom $76(54 \%)$ had Gleason score $3+5$, 46 $(33 \%) 4+4$, and $18(13 \%) 5+3$ disease. Invasive cribriform and/or intraductal carcinoma $(n=87,62 \%)$ was observed more frequently in Gleason score $4+4(93 \%)$ than $3+5(47 \% ; P<0.001)$ and $5+3(44 \% ; P<0.001)$ patients. Gleason pattern 5 was present in 110 (79\%) men: as single cells and/or cords in $99(90 \%)$ and solid fields in 32 (29\%) cases. Solid field pattern 5 coexisted with cribriform architecture $(23 / 32,72 \%)$ more frequently than nonsolid pattern 5 cases $(36 / 78$, $46 \%, P=0.02$ ). In multivariable analysis including age, prostate-specific antigen, pT-stage, surgical margin status, and lymph node metastases, presence of cribriform architecture was an independent parameter for biochemical recurrence-free (hazard ratio (HR) 2.0, 95\% confidence interval (CI) $1.0-3.7 ; P=0.04$ ) and metastasis-free (HR 3.5, 95\% CI 1.0-12.3; $P=$ $0.05)$ survival. In conclusion, invasive cribriform and/or intraductal carcinoma occurs more frequently in Gleason score $4+$ 4 prostate cancer patients than in Gleason score $3+5$ and $5+3$, and is an independent parameter for biochemical recurrence and metastasis. Therefore, cribriform architecture has added value in risk stratification of Gleason score 8 prostate cancer patients.
\end{abstract}

\section{Introduction}

The Gleason grading system for prostate cancer is based on classification of histomorphological growth patterns [1]. At the 2014 meeting of the International Society of

Eva Hollemans

e.hollemans@erasmusmc.nl

1 Department of Pathology, Erasmus MC, University Medical Center, Rotterdam, The Netherlands

2 Department of Urology, Erasmus MC, University Medical Center, Rotterdam, The Netherlands

3 Department of Urology, Franciscus Gasthuis \& Vlietland, Rotterdam, The Netherlands

4 Department of Medical Oncology, Leiden University Medical
Urological Pathology (ISUP), consent was reached that a Grade Group should be reported in conjunction with the Gleason score, based on the initial work of Pierorazio et al. which was endorsed by the World Health Organization (WHO) in 2016 [2-4]. The Grade Group system is

Center, Leiden, The Netherlands

5 Department of Urology, Leiden University Medical Center, Leiden, The Netherlands

6 Department of Pathology, Leiden University Medical Center, Leiden, The Netherlands

7 Department of Urology, Netherlands Cancer Institute, Amsterdam, The Netherlands

8 Department of Pathology, Netherlands Cancer Institute, Amsterdam, The Netherlands 
comprehensive and facilitates patient communication as it labels Gleason score 2-6 as Grade Group 1 and emphasizes the important distinction between Gleason score $3+$ $4=7$ (Grade Group 2) and 4+3=7 (Grade Group 3) prostate cancer. Grade Group 4 prostate cancer encompasses Gleason score 8 tumors, including Gleason score 3 $+5,5+3$, and $4+4[5,6]$. However, it is not yet clear whether these three Gleason score 8 subgroups have similar clinical outcome.

The importance of distinguishing individual prostate cancer growth patterns is increasingly being acknowledged. Gleason pattern 4 encompasses four major growth patterns, including poorly formed, fused, glomeruloid and cribriform glands [3]. The clinical relevance of cribriform architecture in prostate cancer has been well established in recent years, as it is associated with biochemical recurrence, metastasis, and disease-specific death [7-13]. Intraductal carcinoma is characterized by a proliferation of malignant epithelial cells with cribriform or solid architecture distending preexistent acini and prostatic ducts with preservation of basal cells [3]. Although not incorporated in the Gleason score or Grade Group, intraductal carcinoma is independently associated with adverse oncological outcome [8, 14, 15]. The adverse impact of invasive cribriform and intraductal carcinoma has mainly been studied in Gleason score $3+$ 4 prostate cancer, as it might affect clinical decisionmaking in this patient population in particular. Some studies indicate that presence of invasive cribriform and intraductal carcinoma also has independent predictive value in Gleason score 8 prostate cancer patients $[14,16]$.

While the impact of cribriform architecture is well recognized, little is known about the clinical relevance of individual Gleason 5 growth patterns [7]. Gleason pattern 5 encompasses tumor growth in single cells, cords, and solid fields [3]. Furthermore, presence of comedonecrosis is considered Gleason pattern 5, whether it is present within papillary, cribriform, or solid fields. Of notice, recent studies have shown that comedonecrosis more commonly occurs in intraductal carcinoma than in invasive carcinoma, requiring basal cell immunohistochemistry for their distinction [17-19]. While Gleason score 8 prostate cancer is generally considered a high-risk disease requiring immediate therapeutic intervention, analysis of individual Gleason 4 and 5 growth patterns might attribute to risk stratification and optimize personalized treatment decisions. The objective of this study is to compare the clinical characteristics and outcome of Gleason score $3+5,5+3$, and $4+4$ subgroups and to investigate the impact of invasive cribriform and/or intraductal carcinoma in Gleason score 8 radical prostatectomy specimens.

\section{Methods}

\section{Patient selection}

Patients who had undergone radical prostatectomy for prostatic adenocarcinoma from three university medical centers in The Netherlands between 2000 and 2017 were included in this study; 854 patients were operated at Erasmus MC, University Medical Center, Rotterdam; 96 at Leiden University Medical Center (LUMC), Leiden; and 137 at Antoni van Leeuwenhoek Hospital, the Netherlands Cancer Institute (NKI), Amsterdam. Whereas the radical prostatectomies from Erasmus MC were consecutive, those from LUMC and NKI were selected for presence of Gleason score $4+3$ to 10 in the original pathology report. We excluded men who had undergone hormonal, radiation, and/ or viral therapy $(n=23)$ prior to operation [20]. Radical prostatectomy specimens were fixed in neutral-buffered formalin, after which they were sectioned transversely and embedded entirely for diagnostic purposes. All slides were available for pathology review. This study was approved by the institutional Medical Research Ethics Committee (MEC-2018-1614).

\section{Pathologic evaluation}

All 1064 radical prostatectomy specimens were reviewed in common sessions by two investigators ( $\mathrm{EH}, \mathrm{GvL})$, blinded to clinical outcome. For each specimen the following features were recorded: Gleason score and Grade Group according to the 2014 ISUP/2016 WHO guidelines, pTstage according to the American Joint Committee on Cancer TNM 8th edition, surgical margin status, presence of intraductal carcinoma, and percent Gleason 4 and 5 growth patterns $[3,21]$. In case of multifocality we only monitored the characteristics of the index tumor defined as the tumor with the highest grade, stage or volume.

The following Gleason 4 growth patterns were recognized: poorly formed, fused, glomeruloid, and cribriform glands [2, 3]. Furthermore, we distinguished small and large cribriform gland architecture (Fig. 1a, b), since the latter is associated with more aggressive behavior [12]. Large cribriform structures were defined as having a diameter more than twice the size of adjacent benign glands. We examined the following Gleason 5 growth patterns: single cells, cords, and solid fields (Fig. 1c-f). Single cells and cords were grouped for analysis. Solid fields were divided into those with small solid nests containing 10-30 cells, and those consisting of medium to large solid fields with more than 30 cells. In case comedonecrosis was present in invasive cribriform or solid fields, this was considered Gleason pattern 5. Invasive cribriform Gleason pattern 4 and solid pattern 5 either with or without comedonecrosis were 
Fig. 1 Gleason pattern 4 and pattern 5 tumor morphology. a Gleason pattern 4, small invasive cribriform structures, $15 \times$. b Gleason pattern 4 , large invasive cribriform structures, 10x. c Gleason pattern 5, cords, 20x. d Gleason pattern 5, small solid nests with subtle intervening stroma, 20x. e Gleason pattern 5, medium to large sized solid fields, $15 x$

f Gleason pattern 5 , comedonecrosis in a solid field, $15 \times$
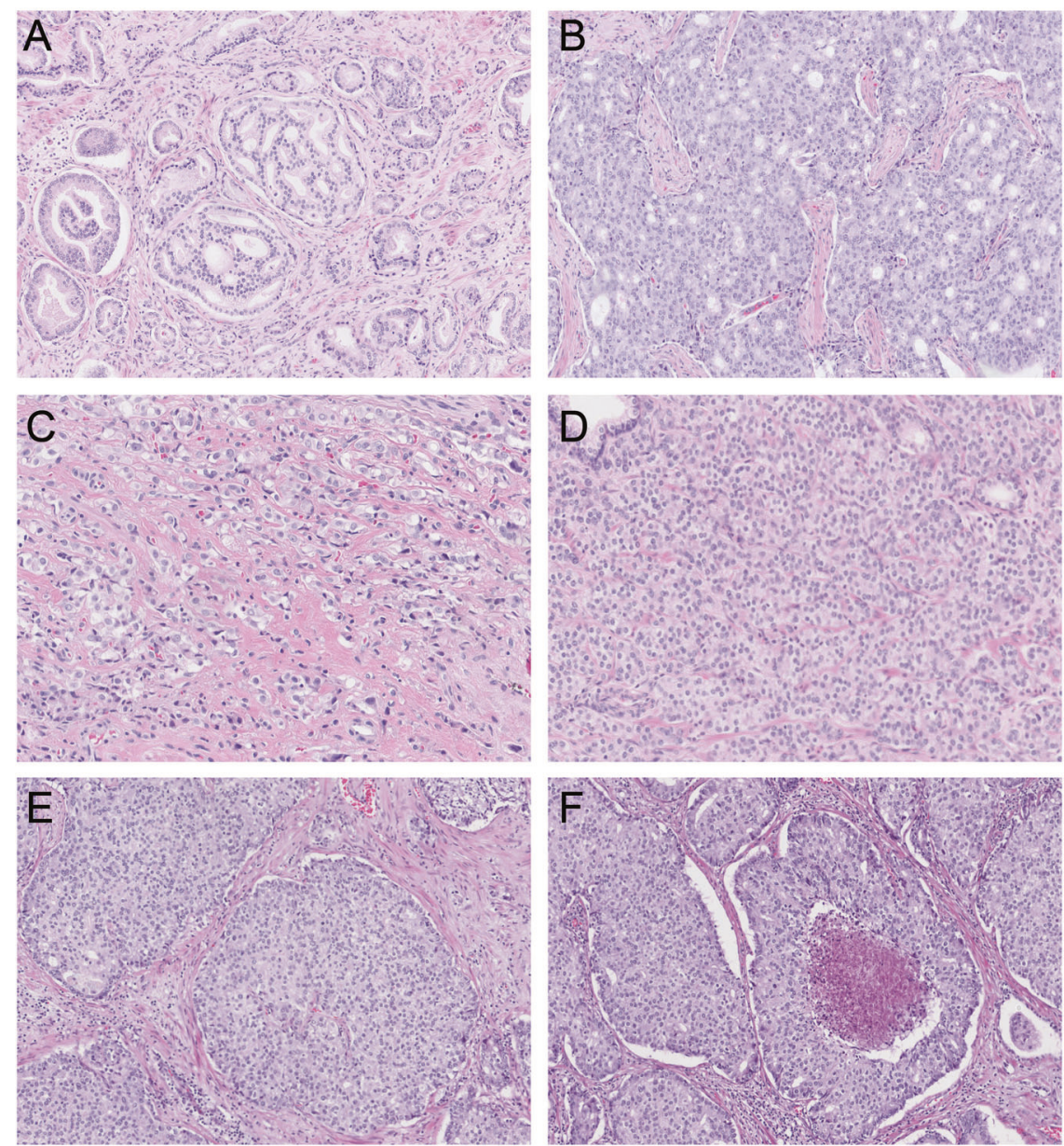

morphologically distinguished from intraductal carcinoma based on the following features: invasive cribriform and solid prostate cancer had irregular borders or formed interconnecting fields, well exceeding the outline of distended preexistent glands, or extended into periprostatic adipose tissue, ejaculatory ducts or seminal vesicles. Intraductal carcinoma was continuous with preexistent glands lined by basal cells, or contained corpora amylacea. In case invasive cribriform or solid carcinoma and intraductal carcinoma could not be differentiated by morphological criteria alone, additional basal cell immunohistochemistry was performed. Basal cell immunohistochemistry (34BE12) was performed in 189/854 (22\%) radical prostatectomy specimens from Erasmus MC, including 14/31 (45\%) Gleason score 8 tumors with cribriform or solid architecture; no paraffin blocks were available from the other hospitals. If basal cells were completely absent, the lesion was classified as either invasive cribriform Gleason pattern 4 or solid pattern 5 carcinoma. When sporadic, scattered or continuous basal cells were identified, the lesion was considered intraductal carcinoma. Intraductal carcinoma and tertiary patterns were not incorporated in the Gleason score $[2,3,22]$. Minor high-grade components occupying $<5 \%$ of the tumor volume were considered as tertiary pattern. The Grade Group concordance rate at revision was 88/135 $(65 \%)$ for radical prostatectomies from NKI and 39/94 (41\%) for specimens from LUMC.

\section{Clinical follow-up}

Clinical follow-up after radical prostatectomy consisted of 6 monthly, and later annual monitoring of serum prostatespecific antigen (PSA) levels. Biochemical recurrence was defined as PSA levels $\geq 0.2 \mathrm{ng} / \mathrm{ml}$ measured at two consecutive points in time, at least 3 months apart with undetectable PSA levels after operation, or as PSA increase of $>2.0 \mathrm{ng} / \mathrm{ml}$ when serum PSA had not declined to zero after operation. Postoperative lymph node and distant metastases were confirmed by biopsy or multidisciplinary consensus.

\section{Statistical analysis}

Continuous variables with normal distribution were analyzed using the independent sample Student's $t$ test for two groups, or one-way ANOVA for $\geq 3$ groups. Variables without normal distribution were analyzed using the 
Mann-Whitney $U$ test for two groups, or Kruskal-Wallis test for $\geq 3$ groups. For comparison of categorical parameters Pearson's chi squared $\left(\chi^{2}\right)$ test was used, and Fisher's exact test in case of small numbers $(n \leq 20)$. Missing PSA values $(n=27)$ were imputed using the median PSA value. Biochemical recurrence-free survival and metastasis-free survival were analyzed using Cox proportional hazards model and visualized by Kaplan-Meier curves. Statistics were performed using SPSS version 24 (IBM, Chicago, IL, USA). Results were considered significant when the twosided $P$ value was $<0.05$.

\section{Results}

\section{Characteristics of Gleason score 8 prostate cancer patients}

Out of 1064 radical prostatectomy specimens, 140 (13\%) had Gleason score 8 prostate cancer. The median age of Gleason score 8 patients was 65.3 years (interquartile range (IQR) 61.4-68.5 years) and median serum PSA level was $10.0 \mathrm{ng} / \mathrm{ml}$ (IQR $7.2-16.0 \mathrm{ng} / \mathrm{ml}$ ). Gleason scores were distributed as follows: $76(54 \%)$ men had Gleason score 3 $+5,46(33 \%)$ Gleason score $4+4$, and $18(13 \%)$ Gleason score $5+3$. Pathologic tumor stage was T2 in $67(48 \%)$ men, T3a in $44(31 \%)$, and T3b in $28(20 \%)$. One (1\%) patient had a T4 tumor and was grouped with T3b tumors for further analysis. Positive surgical margins were present in $68(49 \%)$ cases. Pelvic lymph node dissection was performed in $91(65 \%)$ men, 12 (9\%) of whom had lymph node metastasis. Median follow-up time was 68.7 months (IQR 36.7-102.8).

\section{Clinicopathological features and outcome of Gleason score $3+5,4+4$, and $5+3$}

The clinicopathological features of Gleason score 8 patients stratified for Gleason score are shown in Table 1. The median PSA level of patients with Gleason score $5+3$ prostate cancer was $13.4 \mathrm{ng} / \mathrm{ml}$ (IQR $8.8-26.8 \mathrm{ng} / \mathrm{ml}$ ), significantly higher than for men with Gleason score $3+5$ $(10.0 \mathrm{ng} / \mathrm{ml} ; \mathrm{IQR} 7.4-15.0 \mathrm{ng} / \mathrm{ml} ; P=0.05)$ and Gleason score $4+4(8.9 \mathrm{ng} / \mathrm{ml}$; IQR $6.9-16.0 \mathrm{ng} / \mathrm{ml} ; P=0.03)$. PSA levels of Gleason score $3+5$ and $4+4$ were comparable $(P=0.45)$. Age, pT-stage, surgical margin status and lymph node metastases were not significantly different between groups. While Gleason pattern 4 constituted $\geq 95 \%$ of the tumor volume in Gleason score $4+4$ by definition, it was present in 73/76 (96\%) Gleason score $3+5$ and 12/18
Table 1 Gleason score 8 patients stratified for individual Gleason score (GS).

\begin{tabular}{|c|c|c|c|c|c|}
\hline & $\begin{array}{l}\text { All } \\
n=140\end{array}$ & $\begin{array}{c}\text { GS } 3+5 \\
n=76\end{array}$ & $\begin{array}{c}\text { GS } 4+4 \\
n=46\end{array}$ & $\begin{array}{c}\text { GS } 5+3 \\
n=18\end{array}$ & $P$ value \\
\hline Age (years) & $\begin{array}{l}64.7(65.3 ; \\
61.4-68.5)\end{array}$ & $\begin{array}{l}64.3(64.5 \\
60.7-68.2)\end{array}$ & $\begin{array}{l}65.6(66.2 ; \\
62.4-69.2)\end{array}$ & $\begin{array}{l}64.5(65.2 \\
60.4-67.2)\end{array}$ & 0.46 \\
\hline PSA (ng/ml) & $\begin{array}{l}12.9(10.0 \\
7.2-16.0)\end{array}$ & $\begin{array}{l}12.5(10.0 \\
7.4-15.0)\end{array}$ & $\begin{array}{l}11.5(8.9 \\
6.9-16.0)\end{array}$ & $\begin{array}{l}18.5(13.4 \\
8.8-26.8)\end{array}$ & 0.07 \\
\hline \multicolumn{6}{|l|}{ pT-stage } \\
\hline $\mathrm{T} 2$ & $67(48 \%)$ & $41(54 \%)$ & $19(41 \%)$ & $7(39 \%)$ & 0.35 \\
\hline T3a & $44(31 \%)$ & $19(25 \%)$ & $19(41 \%)$ & $6(33 \%)$ & \\
\hline $\mathrm{T} 3 \mathrm{~b} / \mathrm{T} 4$ & $29(21 \%)$ & $16(21 \%)$ & $8(18 \%)$ & $5(28 \%)$ & \\
\hline Overall cribriform & $87(62 \%)$ & $36(47 \%)$ & $43(93 \%)$ & $8(44 \%)$ & $<0.001$ \\
\hline \multicolumn{6}{|l|}{ Gleason pattern 4} \\
\hline Small cribriform & $83(59 \%)$ & $33(43 \%)$ & $42(91 \%)$ & $8(44 \%)$ & $<0.001$ \\
\hline Large cribriform & $38(27 \%)$ & $6(8 \%)$ & $28(61 \%)$ & $4(22 \%)$ & $<0.001$ \\
\hline Intraductal carcinoma & $48(34 \%)$ & $21(28 \%)$ & $23(50 \%)$ & $4(22 \%)$ & 0.02 \\
\hline \multicolumn{6}{|l|}{ Gleason pattern 5} \\
\hline Single cells and/or cords & $99(71 \%)$ & $72(95 \%)$ & $10(22 \%)$ & $17(94 \%)$ & $<0.001$ \\
\hline Small solid nests & $23(16 \%)$ & $16(21 \%)$ & $1(2 \%)$ & $6(33 \%)$ & 0.003 \\
\hline $\begin{array}{l}\text { Medium to large } \\
\text { solid fields }\end{array}$ & $15(11 \%)$ & $4(5 \%)$ & $5(11 \%)$ & $6(33 \%)$ & 0.006 \\
\hline Comedonecrosis & $9(6 \%)$ & $2(3 \%)$ & $7(15 \%)$ & 0 & 0.02 \\
\hline Positive surgical margins & $68(49 \%)$ & $40(53 \%)$ & $20(44 \%)$ & $8(44 \%)$ & 0.58 \\
\hline $\begin{array}{l}\text { Pelvic lymph node } \\
\text { dissection }\end{array}$ & $91(65 \%)$ & $47(62 \%)$ & $29(63 \%)$ & $15(83 \%)$ & 0.22 \\
\hline Lymph node metastasis & $12(13 \%)$ & $5(11 \%)$ & $4(14 \%)$ & $3(20 \%)$ & 0.54 \\
\hline Biochemical recurrence & $68(49 \%)$ & $31(41 \%)$ & $29(63 \%)$ & $8(44 \%)$ & 0.05 \\
\hline Metastasis & $36(26 \%)$ & $14(18 \%)$ & $18(39 \%)$ & $4(22 \%)$ & 0.04 \\
\hline Disease-specific death & $12(9 \%)$ & $4(5 \%)$ & $7(15 \%)$ & $1(6 \%)$ & 0.17 \\
\hline
\end{tabular}

Values denote either mean (median; IQR) or $n(\%)$.

PSA prostate-specific antigen. 
A

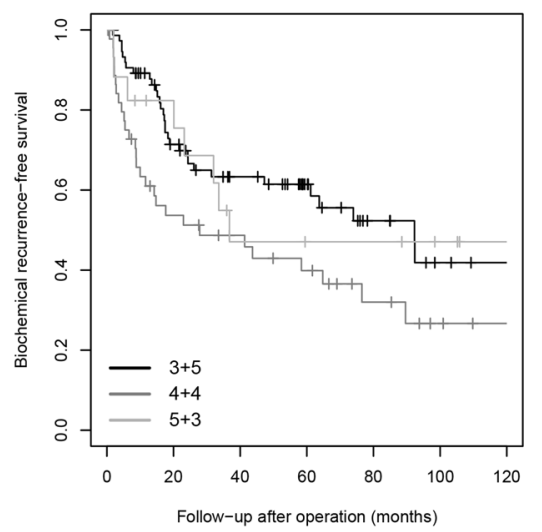

B

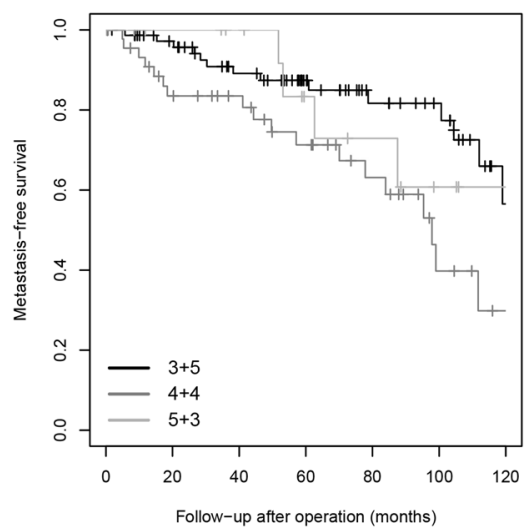

C

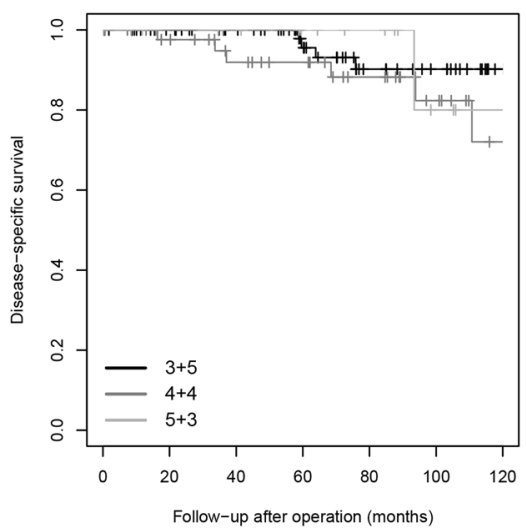

Fig. 2 Survival curves stratified for individual Gleason score. Kaplan-Meier curves of a biochemical recurrence-free survival (log rank $P=$ 0.001 ), b metastasis-free survival ( $\log \operatorname{rank} P<0.001)$, and $\mathbf{c}$ disease-specific survival $(\log \operatorname{rank} P=0.01)$ in Gleason score 8 patients stratified for individual Gleason score.

(67\%) $5+3$ tumors. The median percentage of Gleason pattern 4 was $30 \%$ (IQR $20-35 \%$ ) in $3+5$ tumors and $18 \%$ (IQR $0-21 \%)$ in $5+3$ tumors $(P<0.001)$. Tertiary $(<5 \%)$ Gleason pattern 5 was observed in $16 / 46(35 \%)$ Gleason score $4+4$ tumors.

Biochemical recurrence and postoperative distant metastasis were observed in 68 (49\%) and $36(26 \%)$ patients, respectively. Twenty-nine (63\%) men with Gleason score $4+4$ tumors experienced biochemical recurrence compared to $31(41 \%, P=0.02)$ with Gleason score $3+5$ and $8(44 \%, P=0.78)$ with $5+3$. Biochemical recurrencefree survival was significantly shorter for patients with Gleason score $4+4$ than Gleason score $3+5$ (log rank $P=0.02$ ) prostate cancer. Gleason score $5+3$ had the lowest absolute number of events and did not significantly differ from Gleason score $3+5(\log$ rank $P=0.82)$ and Gleason score $4+4(\log \operatorname{rank} P=0.26$, Fig. 2). A similar trend was found for postoperative metastasis. Metastases occurred in $18(39 \%)$ men with Gleason score $4+4$ compared with $14(18 \%, P=0.01)$ with Gleason score $3+5$ and $4(22 \%, P=0.20)$ men with Gleason score $5+3$. Metastasis-free survival was significantly shorter for patients with Gleason score $4+4$ than Gleason score $3+5$ (log rank $P=0.006$ ) prostate cancer. Gleason score $5+3$ did not significantly differ from Gleason score $3+5$ (log rank $P=0.63)$ and Gleason score $4+4(\log \operatorname{rank} P=0.25)$. The number of disease-specific deaths $(n=12,9 \%)$ was too low for subgroup analysis.

\section{Cribriform architecture in Gleason score 8 prostate cancer}

Invasive and/or intraductal cribriform carcinoma was present in $87(62 \%)$ men, of whom $36(41 \%)$ had Gleason score $3+5$, 43 (49\%) Gleason score $4+4$, and 8 (10\%)
Gleason score $5+3$ tumors. Of these, 83 (95\%) had invasive and $48(55 \%)$ had intraductal cribriform carcinoma. Both patterns were concurrently present in $44(51 \%)$ men. Invasive cribriform carcinoma only was seen in $39(44 \%)$ men and intraductal cribriform carcinoma only in $4(5 \%)$ men. Large cribriform carcinoma was present in 37 (43\%) men with cribriform architecture and was always accompanied by small cribriform carcinoma. Invasive and/or intraductal cribriform carcinoma was observed more frequently in Gleason score $4+4$ than in Gleason score $3+5$ (93\% versus $47 \%, P<0.001)$ and $5+3(93 \%$ versus $44 \%$, $P<0.001)$ tumors. Large invasive cribriform carcinoma also occurred more often in Gleason score $4+4$ than in $3+5(61 \%$ versus $8 \%, P<0.001)$ or $5+3(61 \%$ versus $22 \%, P<0.001)$ tumors, while its appearance in Gleason score $3+5$ and $5+3$ was not significantly different $(P=0.08)$ in this cohort.

Gleason score 8 prostate cancer was stratified based on presence of invasive and/or intraductal cribriform carcinoma (Table 2). Non-organ confined disease (63\% versus $34 \%$, $\geq$ pT3a, $P=0.003$ ) and positive pelvic lymph nodes $(19 \%$ versus $3 \%, P=0.05)$ were more common in patients with cribriform architecture. Age, PSA levels, and surgical margin status were not significantly different between Gleason score 8 patients with or without cribriform architecture. Patients with cribriform architecture had significantly shorter biochemical recurrence-free ( $\log \operatorname{rank} P=$ 0.001 ), metastasis-free (log rank $P<0.001)$, and disease specific (log rank $P=0.01$ ) survival than those without (Fig. 3).

\section{Histomorphology of Gleason pattern 5}

Gleason pattern 5 was observed in 110 (79\%) Gleason score 8 tumors. In addition to men with Gleason score $3+5$ and 
Table 2 Gleason score 8 patients stratified for presence of cribriform architecture.

\begin{tabular}{|c|c|c|c|}
\hline & $\begin{array}{c}\text { Cribriform } \\
\text { negative } \\
n=53\end{array}$ & $\begin{array}{l}\text { Cribriform } \\
\text { positive } \\
n=87\end{array}$ & $P$ value \\
\hline Age (years) & $\begin{array}{l}63.7(64.1 \\
59.7-67.4)\end{array}$ & $\begin{array}{l}65.4(66.1 ; \\
62.7-69.2)\end{array}$ & 0.09 \\
\hline PSA (ng/ml) & $\begin{array}{l}12.5(10.0 \\
7.0-14.0)\end{array}$ & $\begin{array}{l}13.2(10.0 \\
7.5-16.0)\end{array}$ & 0.33 \\
\hline \multicolumn{4}{|l|}{ pT-stage } \\
\hline $\mathrm{T} 2$ & $35(66 \%)$ & $32(37 \%)$ & 0.003 \\
\hline T3a & $10(19 \%)$ & $34(39 \%)$ & \\
\hline $\mathrm{T} 3 \mathrm{~b} / \mathrm{T} 4$ & $8(15 \%)$ & $21(24 \%)$ & \\
\hline Gleason pattern 4 & $45(85 \%)$ & $86(99 \%)$ & $<0.001$ \\
\hline Small cribriform & 0 & $83(95 \%)$ & $<0.001$ \\
\hline Large cribriform & 0 & $38(44 \%)$ & $<0.001$ \\
\hline Intraductal carcinoma & 0 & $48(55 \%)$ & $<0.001$ \\
\hline Gleason pattern 5 & $50(94 \%)$ & $60(69 \%)$ & $<0.001$ \\
\hline $\begin{array}{l}\text { Single cells and/ } \\
\text { or cords }\end{array}$ & $48(91 \%)$ & $51(59 \%)$ & $<0.001$ \\
\hline Small solid nests & $10(19 \%)$ & $13(15 \%)$ & 0.54 \\
\hline $\begin{array}{l}\text { Medium to large } \\
\text { solid fields }\end{array}$ & 0 & $15(17 \%)$ & $<0.001$ \\
\hline Comedonecrosis & 0 & $9(10 \%)$ & 0.02 \\
\hline $\begin{array}{l}\text { Positive surgical } \\
\text { margins }\end{array}$ & $25(47 \%)$ & $43(49 \%)$ & 0.80 \\
\hline $\begin{array}{l}\text { Pelvic lymph node } \\
\text { dissection }\end{array}$ & $32(60 \%)$ & $59(68 \%)$ & 0.37 \\
\hline $\begin{array}{l}\text { Lymph node } \\
\text { metastasis }\end{array}$ & $1(3 \%)$ & $11(19 \%)$ & 0.05 \\
\hline Biochemical recurrence & $16(30 \%)$ & $52(60 \%)$ & 0.001 \\
\hline Metastasis & $4(8 \%)$ & $32(37 \%)$ & $<0.001$ \\
\hline Disease-specific death & 0 & $12(14 \%)$ & 0.004 \\
\hline
\end{tabular}

Values denote either mean (median; IQR) or $n(\%)$.

PSA prostate-specific antigen.

$5+3,16(35 \%)$ men with Gleason score $4+4$ had tertiary Gleason pattern 5. Single cells and/or cords were present in 99/110 (90\%) and solid fields in 32/110 (29\%) tumors. All Gleason 5 patterns were simultaneously present in $26(24 \%)$ cases. Invasive and/or intraductal cribriform carcinoma was present in $23 / 32(72 \%)$ cases with solid pattern 5 and in $36 / 78(46 \%)$ cases with nonsolid pattern 5 $(P=0.02)$. Of interest, the nine solid field cases without associated cribriform architecture all were of the small nested type (Fig. 1d). Comedonecrosis was present in nine cases, 7 (78\%) of which were present in Gleason score $4+4$ tumors. Comedonecrosis was accompanied by cribriform architecture in all 9/9 (100\%) cases and by solid fields in 5/9 (56\%) cases. Moreover, comedonecrosis was observed more often in patients with large cribriform fields $(7 / 20,35 \%)$ than in those without $(2 / 90,2 \%$, $P<0.001)$.

\section{Multivariable analysis of clinical outcome in Gleason score 8 patients}

In univariate Cox regression analysis, pT3a (hazard ratio (HR) 2.1, 95\% confidence interval (CI) $1.1-3.8, P=0.02$ ), pT3b/4 (HR 4.6, 95\% CI 2.5-8.5, $P<0.001$ ), Gleason score $4+4$ (HR $1.9,95 \%$ CI 1.1-3.1, $P=0.02$ ), positive lymph nodes at time of operation (HR 11.8, 95\% CI 5.6-25.2, $P<$ 0.001 ), and overall presence of invasive and/or intraductal cribriform carcinoma (HR 2.4, 95\% CI 1.4-4.1, $P=0.003$ ) were significantly associated with shorter biochemical recurrence-free survival, while age $(P=0.18)$, PSA level $(P=0.43)$, Gleason score $5+3(P=0.78)$ and surgical margin status $(P=0.21)$ were not (Table 3$)$. In multivariable analysis, pT3b/4-stage (HR 4.4, 95\% CI 2.1-9.3, $P<0.001$ ), positive lymph nodes (HR 9.9, 95\% CI 4.2-23.5, $P<0.001$ ), and overall cribriform architecture (HR 2.0, 95\% CI 1.0-3.7, $P=0.04$ ) had independent predictive value for biochemical recurrence-free survival, while Gleason score $4+4$ (HR 1.7, 95\% CI 1.0-2.9, $P=0.07$ ) did not meet conventional measures of significance in this cohort. In case individual cribriform growth patterns were included in multivariable analysis instead of overall cribriform architecture, large invasive cribriform carcinoma (HR $2.0,95 \%$ CI 1.0-4.1, $P=0.05$ ) had independent predictive value for biochemical recurrence-free survival, whereas intraductal cribriform carcinoma (HR 1.3, 95\% CI 0.8-3.5, $P=0.4$ ) and small invasive cribriform carcinoma (HR 1.6, 95\% CI 0.8-3.5, $P=0.2$ ) did not (data not shown).

Similar trends were observed for metastasis as pT3a (HR 2.7, 95\% CI 1.2-6.2, $P=0.02$ ), Gleason score $4+4$ (HR $3.8,95 \%$ CI $1.8-8.1, P=0.001)$, positive lymph nodes (HR 11.5, 95\% CI 5.2-25.9, $P<0.001$ ), and overall cribriform architecture (HR 6.7, 95\% CI 2.0-21.9, $P=0.002$ ) were significantly associated with shorter metastasis-free survival, whereas age $(P=0.80)$, PSA level $(P=0.96)$, pT3b/4 $(P=0.06)$, Gleason score $5+3(P=0.85)$, and positive surgical margins $(P=0.95)$ were not (Table 4$)$. In multivariable analysis, Gleason score $4+4$ (HR 2.4, 95\% CI 1.0-5.9, $P=0.05$ ), positive lymph nodes (HR 15.0, 95\% CI 5.6-40.0, $P<0.001$ ), and overall cribriform architecture (HR 3.5, 95\% CI 1.0-12.3, $P=0.05$ ) had independent predictive value for metastasis-free survival. Due to the low number of events and risk of model overfitting we were not able to include individual cribriform or Gleason 5 growth patterns in multivariable analysis.

\section{Discussion}

Our study demonstrates that among Gleason score 8 prostate cancer patients on radical prostatectomy, biochemical recurrence and metastases occur more often in Gleason 
A

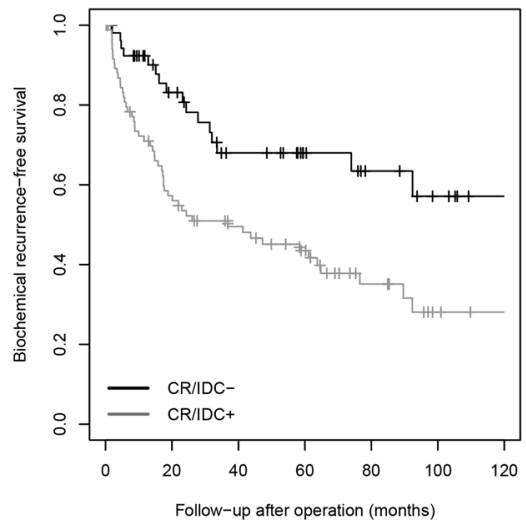

$\mathrm{B}$

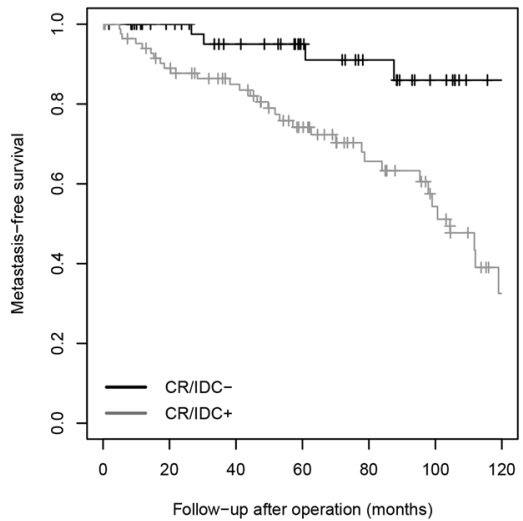

C

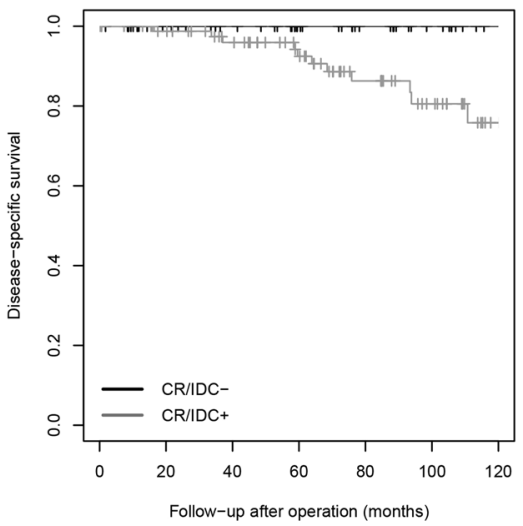

Fig. 3 Survival curves stratified for cribriform architecture. Kaplan-Meier curves of a biochemical recurrence-free survival (log rank $P=0.001$ ), b metastasis-free survival (log rank $P<0.001$ ), and c disease-specific survival $(\log \operatorname{rank} P=0.01)$ in Gleason score 8 patients with invasive and/or intraductal cribriform carcinoma (CR/ IDC + ) and without invasive and/or intraductal cribriform carcinoma (CR/IDC-).
Table 3 Cox regression analysis for biochemical recurrence-free survival in Gleason score 8 patients.

\begin{tabular}{|c|c|c|c|c|c|c|}
\hline & \multicolumn{3}{|c|}{ Univariate } & \multicolumn{3}{|c|}{ Multivariable } \\
\hline & $\mathrm{HR}^{\mathrm{a}}$ & $95 \% \mathrm{CI}$ & $P$ value & HR & $95 \% \mathrm{CI}$ & $P$ value \\
\hline Age (years) & 1.0 & $0.9-1.0$ & 0.18 & 1.0 & $0.9-1.0$ & 0.05 \\
\hline PSA (ng/ml) & 1.0 & $1.0-1.0$ & 0.43 & 1.0 & $1.0-1.0$ & 0.23 \\
\hline \multicolumn{7}{|l|}{ pT-stage } \\
\hline $\mathrm{T} 2$ & ref & & & ref & & \\
\hline T3a & 2.1 & $1.1-3.8$ & 0.02 & 1.8 & $1.0-3.4$ & 0.06 \\
\hline $\mathrm{T} 3 \mathrm{~b} / \mathrm{T} 4$ & 4.6 & $2.5-8.5$ & $<0.001$ & 4.4 & $2.1-9.3$ & $<0.001$ \\
\hline \multicolumn{7}{|l|}{ Gleason score } \\
\hline $3+5$ & $r e f$ & & & $r e f$ & & \\
\hline $4+4$ & 1.9 & $1.1-3.1$ & 0.02 & 1.7 & $1.0-2.9$ & 0.07 \\
\hline $5+3$ & 1.1 & $0.5-2.4$ & 0.78 & 1.5 & $0.6-3.6$ & 0.37 \\
\hline $\begin{array}{l}\text { Positive surgical } \\
\text { margins }\end{array}$ & 1.4 & $0.8-2.2$ & 0.21 & 0.8 & $0.5-1.5$ & 0.55 \\
\hline $\begin{array}{l}\text { Pelvic lymph } \\
\text { node metastasis }\end{array}$ & 11.8 & $5.6-25.2$ & $<0.001$ & 9.9 & $4.2-23.5$ & $<0.001$ \\
\hline $\begin{array}{l}\text { Cribriform } \\
\text { architecture }\end{array}$ & 2.4 & $1.4-4.1$ & 0.003 & 2.0 & $1.0-3.7$ & 0.04 \\
\hline
\end{tabular}

CI confidence interval.

${ }^{\mathrm{a}} \mathrm{HR}=$ hazard ratio.

score $4+4$ than in Gleason score $3+5$ or $5+3$ tumors. Invasive and/or intraductal cribriform carcinoma was observed in $62 \%$ of tumors and was associated with adverse pathological features and clinical outcome. Cribriform architecture occurred more frequently in men with Gleason score $4+4(93 \%)$ than in those with Gleason score $3+5$ $(47 \%)$ and $5+3(44 \%)$. In multivariable analysis, cribriform architecture was an independent parameter for biochemical recurrence- and metastasis-free survival, while
Table 4 Cox regression analysis for metastasis-free survival in Gleason score 8 patients.

\begin{tabular}{|c|c|c|c|c|c|c|}
\hline & \multicolumn{3}{|c|}{ Univariate } & \multicolumn{3}{|c|}{ Multivariable } \\
\hline & $\mathrm{HR}^{\mathrm{a}}$ & $95 \% \mathrm{CI}$ & $P$ value & HR & $95 \% \mathrm{CI}$ & $P$ value \\
\hline Age (years) & 1.0 & $0.9-1.1$ & 0.80 & 1.0 & $0.9-1.1$ & 0.52 \\
\hline PSA (ng/ml) & 1.0 & $1.0-1.0$ & 0.96 & 1.0 & $0.9-1.0$ & 0.50 \\
\hline \multicolumn{7}{|l|}{ pT-stage } \\
\hline $\mathrm{T} 2$ & ref & & & ref & & \\
\hline $\mathrm{T} 3 \mathrm{a}$ & 2.7 & $1.2-6.2$ & 0.02 & 2.5 & $1.0-6.7$ & 0.06 \\
\hline $\mathrm{T} 3 \mathrm{~b} / \mathrm{T} 4$ & 2.4 & $1.0-6.0$ & 0.06 & 1.3 & $0.4-3.8$ & 0.67 \\
\hline \multicolumn{7}{|l|}{ Gleason score } \\
\hline $3+5$ & ref & & & $r e f$ & & \\
\hline $4+4$ & 3.8 & $1.8-8.1$ & 0.001 & 2.4 & $1.0-5.9$ & 0.05 \\
\hline $5+3$ & 1.1 & $0.32-4.0$ & 0.85 & 0.7 & $0.2-3.0$ & 0.65 \\
\hline $\begin{array}{l}\text { Positive } \\
\text { surgical } \\
\text { margins }\end{array}$ & 1.0 & $0.5-2.0$ & 0.95 & 1.1 & $0.5-2.4$ & 0.84 \\
\hline $\begin{array}{l}\text { Pelvic lymph } \\
\text { node metastasis }\end{array}$ & 11.5 & $5.2-25.9$ & $<0.001$ & 15.0 & $5.6-40.0$ & $<0.001$ \\
\hline $\begin{array}{l}\text { Cribriform } \\
\text { architecture }\end{array}$ & 6.7 & $2.0-21.9$ & 0.002 & 3.5 & $1.0-12.3$ & 0.05 \\
\hline
\end{tabular}

CI confidence interval.

${ }^{\mathrm{a}} \mathrm{HR}=$ hazard ratio.

Gleason score was not. Therefore, cribriform architecture also has important value for risk stratification among Gleason score 8 prostate cancer patients.

Since the introduction of Grade Groups several reports have analyzed the clinical outcome of Gleason score $3+5$, $4+4$, and $5+3$ prostate cancer [4]. Some of these studies found that men with Gleason score $3+5$ at radical prostatectomy had reduced risk of biochemical recurrence among Gleason score 8 patients [23, 24]. Others did not find 
a difference among Gleason score 8 subgroups or concluded that men with primary Gleason pattern 5 had worse outcome [16, 25-28]. This variability of results might be explained by the use of different specimen types and clinical outcome measures [29]. Furthermore, Gleason score 8 is relatively uncommon, hampering statistical analysis on large numbers of patients or resulting in clustering of Gleason score $5+3$ and $5+3$ tumors [28, 30]. Finally, from a morphologically point of view, Gleason score 8 prostate cancer is a very heterogeneous disease including highly variable quantities of Gleason 3, 4, and 5 growth patterns. This heterogeneity might lead to significant interobserver variability in tumor grading. For instance, Shah et al. only found fair interobserver reproducibility for Gleason pattern 5 assignment among 16 international expert genitourinary pathologists [31]. Upon rereview of 40 archival cases with Gleason score $5+3$ prostate cancer, Kryvenko et al. assigned the same score in only $4(10 \%)$ specimens, but upgraded $57.5 \%$ and downgraded $17.5 \%$ of cases [32].

Many studies demonstrated worse clinical outcome for patients with cribriform architecture [7-11]. Most of these studies investigated cribriform architecture in intermediate grade prostate cancer, while the impact of cribriform architecture in Gleason score $\geq 4+3$ is less well established. In Gleason score 7-10 prostate cancer biopsy patients, presence of cribriform architecture has been associated with advanced pathological stage and worse disease-specific survival compared with those with cribriform-negative biopsies [14, 33]. Harding-Jackson et al. found cribriform architecture, but not Gleason score, to have independent predictive value for cancer-specific survival in Gleason score 8 patients [16]. In the current study, we confirmed that cribriform architecture had strong discriminative value, even in aggressive Gleason score 8 prostate cancer. Both overall invasive cribriform and/or intraductal carcinoma as well as its more aggressive large cribriform variant were significantly more often observed in Gleason score $4+4$, than $3+5$ and $5+3$ disease. Since its association with adverse outcome, the high frequency of cribriform architecture might well explain the worse outcome of Gleason score $4+4$ prostate cancer compared with those with $3+5$ in our study, while the low number of $5+3$ patients hampered powerful statistical analysis. Of interest, however, is that in multivariable analysis not only cribriform architecture but also Gleason score $4+4$ had independent prognostic value for metastasis-free survival. A similar trend was observed for biochemical recurrence-free survival although the predictive value of Gleason score $4+4$ did not reach conventional measures of significance $(P=0.07)$. This implicates that other grading factors apart from cribriform architecture contribute to the worse outcome in Gleason score $4+4$ patients. A possible explanation could be that Gleason score $4+4$ disease has the lowest percent of Gleason 3 growth pattern, which is by definition present in less than $5 \%$ of the tumor volume. In $3+5$ disease, percent Gleason pattern 3 theoretically varies from $50 \%$ to $95 \%$, while it occupies $5 \%$ to $50 \%$ in Gleason score $5+3$ tumors. Some groups have shown independent prognostic value for percent Gleason pattern 4 and 5, which outperformed Gleason score [34]. The inverse could well be true for percent Gleason pattern 3; if a tumor still has the biological capacity to mature into well-delineated glandular structures it is associated with better outcome.

Little is known about the predictive value of individual Gleason grade 5 growth patterns, which have been reported as either single cells, cords, small solid cylinders, solid fields, and presence of comedonecrosis [3]. Single cells and/ or cords are the most common Gleason pattern $5[35,36]$. Flood et al. found that presence of solid fields and number of different Gleason 5 growth patterns were associated with shorter biochemical recurrence-free survival in Gleason score 9-10 prostatectomies [35]. Compared with other Gleason 5 patterns, comedonecrosis was associated with non-organ confined disease and biochemical recurrence [19, 37]. While individual Gleason 4 growth patterns have increasingly been subject to clinicopathological analysis, information on the clinical relevance of Gleason 5 patterns is still scarce. Our group recently performed in-depth threedimensional visualization of prostate adenocarcinoma architectural growth patterns and revealed two separate morphological groups [38]. The first group consists of a tubular network in which the vast majority if not all tumor cells are in direct contact with surrounding stroma. This group encompasses the morphological continuum of Gleason pattern 3 , poorly formed and fused pattern 4 , and single cells and cords pattern 5 . The second group has contiguous epithelial proliferations in which the majority of tumor cells are not in contact with surrounding stroma and consists of cribriform pattern 4 and solid pattern 5 with or without comedonecrosis. Our current finding that solid fields mostly coexisted with cribriform structures is reflective of this continuum. In the current study, we distinguished between small nested cylinders consisting up to 30 tumor cells and larger solid fields. While the latter was continuous with cribriform growth, small nested cylinders were not. This suggests that both have different biological and possibly clinical relevance. However, larger studies are required to perform statistical analysis on the clinical relevance of individual Gleason 5 growth patterns.

Strong points of this study are the detailed histological review of radical prostatectomy specimens and the classification of cribriform architecture with the use of strict morphological criteria and additional immunohistochemistry. The study is limited by the retrospective study design. The inclusion of high-grade samples from two participating 
centers could have resulted in a selection bias. Furthermore, the relatively short follow-up of 59 months and limited number of patients restricted robust statistical analysis.

In conclusion, Gleason score 8 is a heterogeneous group of prostate cancers. Although clinicopathological characteristics of Gleason score $3+5,4+4$, and $5+3$ are mostly similar, Gleason score $4+4$ patients have a higher risk of adverse events. Cribriform architecture is an independent predictor for metastasis-free survival and has better discriminative value for clinicopathological outcome than Gleason score. Therefore, reporting cribriform architecture might add value in risk stratification of Gleason score 8 prostate cancer patients.

Acknowledgements This research was supported by a grant from the Jaap Schouten Foundation.

\section{Compliance with ethical standards}

Conflict of interest The authors declare that they have no conflict of interest.

Publisher's note Springer Nature remains neutral with regard to jurisdictional claims in published maps and institutional affiliations.

Open Access This article is licensed under a Creative Commons Attribution 4.0 International License, which permits use, sharing, adaptation, distribution and reproduction in any medium or format, as long as you give appropriate credit to the original author(s) and the source, provide a link to the Creative Commons license, and indicate if changes were made. The images or other third party material in this article are included in the article's Creative Commons license, unless indicated otherwise in a credit line to the material. If material is not included in the article's Creative Commons license and your intended use is not permitted by statutory regulation or exceeds the permitted use, you will need to obtain permission directly from the copyright holder. To view a copy of this license, visit http://creativecommons. org/licenses/by/4.0/.

\section{References}

1. Gleason DF. Classification of prostatic carcinomas. Cancer Chemother Rep. 1966;50:125-8.

2. Humphrey PA, Moch H, Cubilla AL, Ulbright TM, Reuter VE. The 2016 WHO classification of tumours of the urinary system and male genital organs-part B: prostate and bladder tumours. Eur Urol. 2016;70:106-19.

3. Epstein JI, Egevad L, Amin MB, Delahunt B, Srigley JR, Humphrey PA, et al. The 2014 International Society of Urological Pathology (ISUP) consensus conference on gleason grading of prostatic carcinoma: definition of grading patterns and proposal for a new grading system. Am J Surg Pathol. 2016; 40:244-52.

4. Pierorazio PM, Walsh PC, Partin AW, Epstein JI. Prognostic Gleason grade grouping: data based on the modified Gleason scoring system. BJU Int. 2013;111:753-60.

5. Herget KA, Patel DP, Hanson HA, Sweeney C, Lowrance WT. Recent decline in prostate cancer incidence in the United States, by age, stage, and Gleason score. Cancer Med. 2016;5:136-41.
6. Epstein JI, Feng Z, Trock BJ, Pierorazio PM. Upgrading and downgrading of prostate cancer from biopsy to radical prostatectomy: incidence and predictive factors using the modified Gleason grading system and factoring in tertiary grades. Eur Urol. 2012;61:1019-24.

7. Iczkowski KA, Torkko KC, Kotnis GR, Wilson RS, Huang W, Wheeler TM, et al. Digital quantification of five high-grade prostate cancer patterns, including the cribriform pattern, and their association with adverse outcome. Am J Clin Pathol. 2011;136: 98-107.

8. Kimura K, Tsuzuki T, Kato M, Saito AM, Sassa N, Ishida R, et al. Prognostic value of intraductal carcinoma of the prostate in radical prostatectomy specimens. Prostate. 2014;74:680-7.

9. Kir G, Sarbay BC, Gumus E, Topal CS. The association of the cribriform pattern with outcome for prostatic adenocarcinomas. Pathol Res Pract. 2014;210:640-4.

10. Kweldam CF, Wildhagen MF, Steyerberg EW, Bangma $\mathrm{CH}$, van der Kwast TH, van Leenders GJ. Cribriform growth is highly predictive for postoperative metastasis and disease-specific death in Gleason score 7 prostate cancer. Mod Pathol. 2015;28:457-64.

11. Trudel D, Downes MR, Sykes J, Kron KJ, Trachtenberg J, van der Kwast TH. Prognostic impact of intraductal carcinoma and large cribriform carcinoma architecture after prostatectomy in a contemporary cohort. Eur J Cancer. 2014;50:1610-6.

12. Hollemans E, Verhoef EI, Bangma CH, Rietbergen J, Helleman J, Roobol MJ, et al. Large cribriform growth pattern identifies ISUP grade 2 prostate cancer at high risk for recurrence and metastasis. Mod Pathol. 2019;32:139-46.

13. Zhang X RLL, Cheville J. Gleason Grade 4 expansile cribriform pattern is associated with poor prognosis in prostate cancer. In: The 107th Annual Meeting of the United States and Canadian Academy of Pathology. Vancouver, BC, Canada: USCAP; 2018. abstract nr. 114.

14. Kweldam CF, Kummerlin IP, Nieboer D, Verhoef EI, Steyerberg EW, van der Kwast TH, et al. Disease-specific survival of patients with invasive cribriform and intraductal prostate cancer at diagnostic biopsy. Mod Pathol. 2016;29:630-6.

15. Robinson B, Magi-Galluzzi C, Zhou M. Intraductal carcinoma of the prostate. Arch Pathol Lab Med. 2012;136:418-25.

16. Harding-Jackson N, Kryvenko ON, Whittington EE, Eastwood DC, Tjionas GA, Jorda M, et al. Outcome of Gleason $3+5=8$ prostate cancer diagnosed on needle biopsy: prognostic comparison with Gleason 4+4=8. J Urol. 2016;196:1076-81.

17. Fine SW, Al-Ahmadie HA, Chen YB, Gopalan A, Tickoo SK, Reuter VE. Comedonecrosis revisited: strong association with intraductal carcinoma of the prostate. Am J Surg Pathol. 2018;42:1036-41.

18. Madan R, Deebajah M, Alanee S, Gupta NS, Carskadon S, Palanisamy N, et al. Prostate cancer with comedonecrosis is frequently, but not exclusively, intraductal carcinoma: a need for reappraisal of grading criteria. Histopathology. 2019;74:1081-7.

19. Acosta AM, Vormittag E, Al Rasheed MRH, Sharif A, Mon KS, Kajdacsy-Balla A, et al. Comparison of prostatic adenocarcinoma Gleason 5 and intraductal carcinoma of the prostate with tumor necrosis. A morphometric study. Pathol Res Pract. 2018;214: 1681-5.

20. van der Linden RR, Haagmans BL, Mongiat-Artus P, van Doornum GJ, Kraaij R, Kadmon D, et al. Virus specific immune responses after human neoadjuvant adenovirus-mediated suicide gene therapy for prostate cancer. Eur Urol. 2005;48:153-61.

21. Buyyounouski MK, Choyke PL, McKenney JK, Sartor O, Sandler $\mathrm{HM}$, Amin MB, et al. Prostate cancer-major changes in the American Joint Committee on Cancer eighth edition cancer staging manual. CA Cancer J Clin. 2017;67:245-53.

22. Epstein JI, Amin MB, Reuter VE, Humphrey PA. Contemporary Gleason grading of prostatic carcinoma: an update with discussion 
on practical issues to implement the 2014 International Society of Urological Pathology (ISUP) consensus conference on gleason grading of prostatic carcinoma. Am J Surg Pathol. 2017;41:1-7.

23. van den Bergh RC, van der Kwast TH, de Jong J, Zargar H, Ryan AJ, Costello AJ, et al. Validation of the novel International Society of Urological Pathology 2014 five-tier Gleason grade grouping: biochemical recurrence rates for $3+5$ disease may be overestimated. BJU Int. 2016;118:502-5.

24. Gandaglia G, Karnes RJ, Sivaraman A, Moschini M, Fossati N, Zaffuto $\mathrm{E}$, et al. Are all grade group 4 prostate cancers created equal? Implications for the applicability of the novel grade grouping. Urol Oncol. 2017;35:461.e467-14.

25. Mahal BA, Muralidhar V, Chen YW, Choueiri TK, Hoffman KE, $\mathrm{Hu} \mathrm{JC}$, et al. Gleason score $5+3=8$ prostate cancer: much more like Gleason score 9? BJU Int. 2016;118:95-101.

26. Rusthoven CG, Carlson JA, Waxweiler TV, Yeh N, Raben D, Flaig TW, et al. The prognostic significance of Gleason scores in metastatic prostate cancer. Urol Oncol. 2014;32:707-13.

27. Rusthoven CG, Waxweiler TV, DeWitt PE, Flaig TW, Raben D, Kavanagh BD. Gleason stratifications prognostic for survival in men receiving definitive external beam radiation therapy for localized prostate cancer. Urol Oncol. 2015;33:71.e11-9.

28. Huynh MA, Chen MH, Wu J, Braccioforte MH, Moran BJ, D'Amico AV. Gleason Score $3+5$ or $5+3$ versus $4+4$ prostate cancer: the risk of death. Eur Urol. 2016;69:976-9.

29. Lu TC, Collins L, Cohen P, Jay A, Campbell JM, O'Callaghan M. Prognostic differences in ISUP Grade Group 4: a systematic review and meta-analysis. Pathol Oncol Res. 2020;26:1367-75.

30. Lu TC, Moretti K, Beckmann K, Cohen P, O'Callaghan M. ISUP Group 4-a homogenous group of prostate cancers? Pathol Oncol Res. 2018;24:921-5.
31. Shah RB, Li J, Cheng L, Egevad L, Deng FM, Fine SW, et al. Diagnosis of Gleason pattern 5 prostate adenocarcinoma on core needle biopsy: an interobserver reproducibility study among urologic pathologists. Am J Surg Pathol. 2015;39:1242-9.

32. Kryvenko ON, Williamson SR, Schwartz LE, Epstein JI. Gleason score $5+3=8$ (grade group 4) prostate cancer-a rare occurrence with contemporary grading. Hum Pathol. 2020;97:40-51.

33. Masoomian M, Downes MR, Sweet J, Cheung C, Evans AJ, Fleshner N, et al. Concordance of biopsy and prostatectomy diagnosis of intraductal and cribriform carcinoma in a prospectively collected data set. Histopathology. 2019;74:474-82.

34. Vis AN, Roemeling S, Kranse R, Schroder FH, van der Kwast TH. Should we replace the Gleason score with the amount of high-grade prostate cancer? Eur Urol. 2007;51:931-9.

35. Flood TA, Schieda N, Sim J, Breau RH, Morash C, Belanger EC, et al. Evaluation of tumor morphologies and association with biochemical recurrence after radical prostatectomy in grade group 5 prostate cancer. Virchows Arch. 2018;472:205-12.

36. Gottipati S, Warncke J, Vollmer R, Humphrey PA. Usual and unusual histologic patterns of high Gleason score 8 to 10 adenocarcinoma of the prostate in needle biopsy tissue. Am J Surg Pathol. 2012;36:900-7.

37. Acosta AM, Al Rasheed MRH, Rauscher GH, Vormittag E, Mon KS, Sharif A, et al. Tumor necrosis in radical prostatectomies with high-grade prostate cancer is associated with multiple poor prognostic features and a high prevalence of residual disease. Hum Pathol. 2018;75:1-9.

38. Verhoef EI, van Cappellen WA, Slotman JA, Kremers GJ, EwingGraham PC, Houtsmuller AB, et al. Three-dimensional analysis reveals two major architectural subgroups of prostate cancer growth patterns. Mod Pathol. 2019;32:1032-41. 